\title{
Detection and Classification of Insect Sounds in a Grain Silo using a Neural Network
}

\author{
Kevin M. Coggins \\ University of Florida \\ kcoggins@eel.ufl.edu
}

\author{
Jose Principe, Ph.D. \\ University of Florida \\ principe@cnel.ufl.edu
}

\begin{abstract}
This paper presents the application of a time-delay neural network to the detection and classification of time signatures produced by insect sounds in a stored grain silo. Conventional methods of insect monitoring can only detect some of the adult insects and none of the larvae insects, which are the most destructive to the grain. The acoustic vibrations generated by the adult and larvae when moving or chewing have distinct time signatures. Random grain settling vibrations and external vibrations add noise to the system. A time-delay neural network with feature extraction was successfully trained to distinguish between these four classes of sounds.
\end{abstract}

\section{Introduction}

The agricultural community needs accurate monitoring of stored grain. The major result is to reduce the amount of hazardous chemicals applied to the grain. There is a large interest worldwide to reduce the use of hazardous fumigants, specifically phosphine, hydrocyanic gas and methyl bromide. It is both costly and dangerous to use these chemicals. Phosphine is deadly to humans in concentrations as low as 0.3 PPM. There is also a need to determine the quality of the grain in respect to allowable insect populations. There are currently no accurate methods to monitor insect population in stored grain. The current method is to manually count the number of insects in a given portion and estimate the insect content of the bulk. One experimental method can pick up the insect sounds, but cannot discriminate between larvae, adult, or false sounds [1]. A real-time system that can accurately monitor insect activity within the silo is needed.

Sound is defined as the propagation of a pressure wave through a medium. The medium for sound propagation in our system is the grain, which is a semi-solid medium with air pockets. Sound pressure waves travels further and with less attenuation through solids than through gases. A piezoelectric transducer is used to translate the pressure wave into an electrical signal. Piezoelectric transducers are usually used to convert airborne acoustic waves into electrical signals. The piezoelectric transducers in our system are modified such that the piezoelectric material rests directly against the grain medium, thus allowing us to transduce the pressure waves in the semi-solid grain into electrical signals.

The most destructive grain pest, the rice weevil (Sitophilus oryzae), is the target insect for the neural network. Entomologists rate the rice weevil as the most important grain pest worldwide [2]. The rice weevil is known around the world to infest rye, buckwheat, table beans, stored cotton, grapes, cashew nuts, cereals, and wheat products of every kind. With a life span of 120-150 days, and laying as many as 25 eggs per day, the rice weevil can destroy a great quantity of grain prior to visual detection. The majority of the rice weevil infestations occur in wheat products, therefore wheat is the environment used to detect and classify the insect sounds.

There are four general classes of sound that occur in grain silos: adult sounds, larvae sounds, grain-settling sounds, and external sounds that penetrate the grain mass. Instrumentation noise is always present but is normally a broadband low amplitude signal. The two sounds of interest are the adult and larvae sounds. Adult insects generate sounds by chewing on grain kernels and by moving through the grain. Larvae insects generate sounds by chewing on the grain kernel from the inside. The larvae begins its life cycle on the inside of the grain kernel, where it is deposited by the female adult insect. Visual inspection of grain will not detect larvae. Grain settling sounds are sounds that occur randomly throughout the bulk when individual grains shift due to a propagating vibration or a localized instability. External sounds that occur include machinery vibration and related noises.

This paper presents a neural network to detect and classify the time signatures produced by insects. The neural network is composed of a preprocessor based on principal component analysis (PCA) and a one-hidden layer timedelay neural network (TDNN) trained with 
backpropagation. Eight network configurations are examined, varying the number of processing elements (PEs) in the hidden layer, the PCA configuration, and the number of taps in the TDNN. The detection and classification performance of the eight network configurations is presented in the form of ROCs (receiver operating characteristics) and confusion matrices.

\section{A neural template matcher for time signatures}

Figures 1 through 5 depict the four classes of input data which are sounds caused by larvae, adults, grain settling, and external noises. The sounds can be compared by amplituce, frequency spectrum and duration. The adult insect generates sound by moving through the grain mass and by chewing on grain kernels. The adult movement sound is typically an impulse followed by a steadily decaying sinusoid (Figure 1). The adult chewing sound is higher in frequency but also consists of an impulse followed by a steadily decaying sinusoid (Figure 2). It is very infrequent and will not be considered in our work.

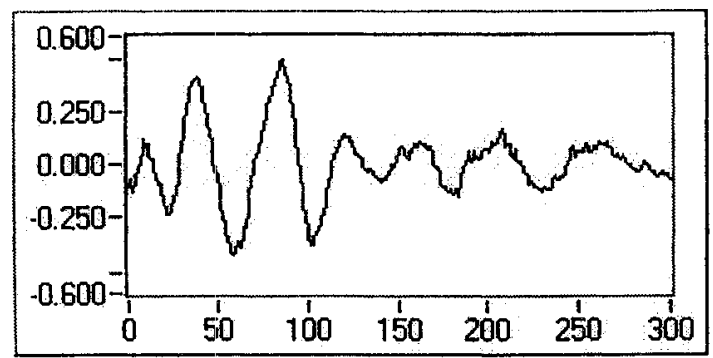

Fig. 1. Adult Insect Movement Sound

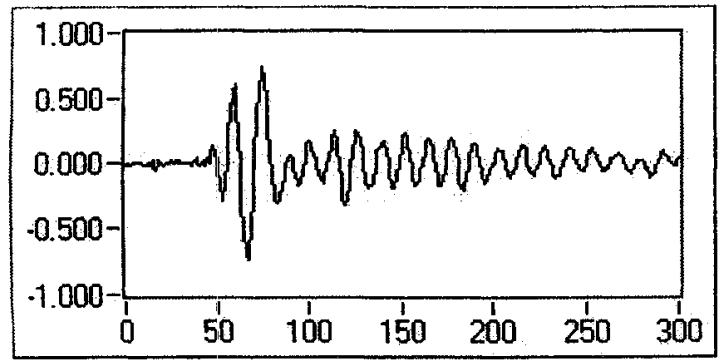

Fig. 2. Adult Insect Chewing Sound

The larvae, being trapped inside the grain kernel, can only generate sounds by chewing. The larvae chewing sound is small in amplitude compared to the adult sounds, yet much higher in frequency (Figure 3). The amplitude of the larvae chewing sounds is close to the noise floor.

The problematic and randomly-occurring grain settling sound consists of a sharp impulse, similar in spectrum to adult sounds, followed by a decaying sinusoid (Figure 4).

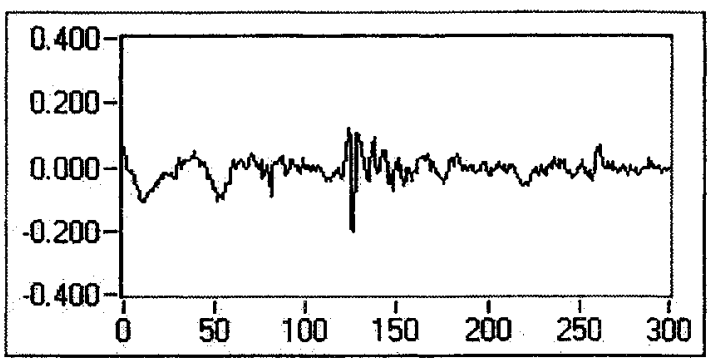

Fig. 3. Larvae Insect Chewing Sound

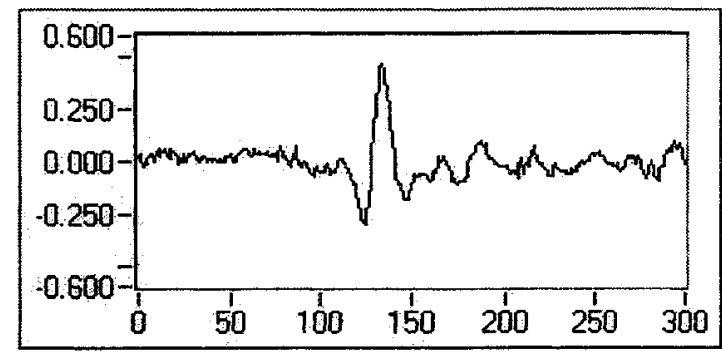

Fig. 4. Grain Settling Sound

External noises that penetrate the grain are often lowfrequency sinusoids from operating machinery. Figure 5 shows a typical external noise.

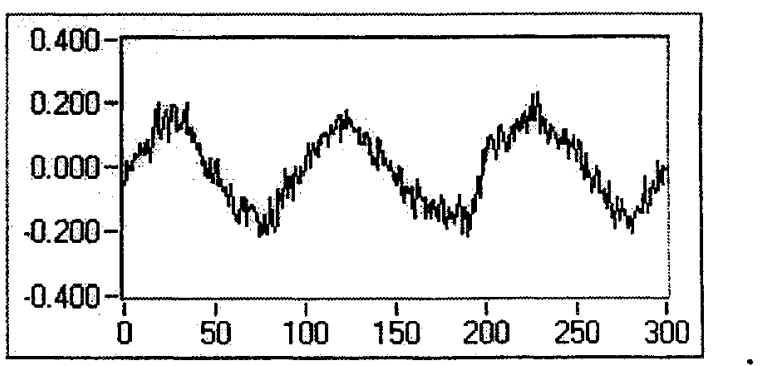

Fig. 5. External Noise Sound

It is obvious that a dynamic network is needed to attack this problem since the information to discriminate these 4 classes of sounds is contained in the history of the time series. The basic idea is to set a window in time that maps the time signature into a reconstruction space where the classification can be achieved. This is basically the same principle used by Waibel for phoneme classification [3]. The big difference is that in our problem the input signal is not segmented as in speech. Abir and Principe [4] proposed a detector for epileptic spikes that did not required pre-segmentation of the input. They designed a desired time series that basically ramps to one at the end of an event of interest, and settles around zero during the background (i.e. when no signature is present).

The function of the neural network is to find the mapping from the waveshape in its time window to the desired 
response, but here the inputs are time signals instead of static patterns. The network is instructed to produce a high response to the time signature and a low response for the background. This function is reminiscent of a matched filter [5]. But instead of implementing the linear operation of convolution, in our case we have the power of arbitrary mappings implemented by the multilayer perceptrons (MLP) [6]. Effectively the neural network becomes a neural template matcher.

\section{Experimental setup}

The experimental setup consists of three components: grain-storage container, data acquisition system, and the neural network (Figure 6). The grain-storage container consists of a cylindrical tube lined with piezoelectric transducers. The cylindrical tube holds exactly $1 \mathrm{~kg}$ of grain. The larvae-infested kernels are placed in positions equidistant to two piezoelectric sensors while the adult insects are allowed to freely roam the grain. Adult insects are generally very active and will generate sounds by moving continuously throughout the bulk of the grain.

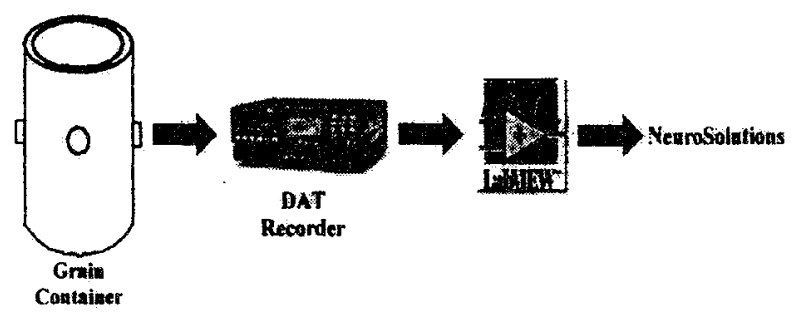

Fig. 6. System Configuration

Each piezoelectric transducer has its own amplification circuitry located directly behind the transducer to reduce signal degradation and noise pickup. The amplifier consists of an instrumentation amplifier followed by a high-gain amplifier. The passband ranges from $1 \mathrm{KHz}$ to $10 \mathrm{KHz}$ with a minimum gain of $80 \mathrm{~dB}$ across the passband. The amplifier signal is then sampled by a SONY PC-216A Digital Audio Tape (DAT) recorder at a sampling rate of $48 \mathrm{kHz}$ with 16-bit quantization. The data is stored on the digital audio tape and can be played back in real time or transferred digitally to a computer as needed. National Instrument's LabView is used to control the DAT recorder and transfer the data to a format useable by the neural network simulator. A Pentium workstation is used to implement the data collection and network simulation.

\section{Implementation}

The commercial simulator NeuroSolutions [7] is used to implement and train the neural network. The general architecture of the neural network is shown in Figure 7.

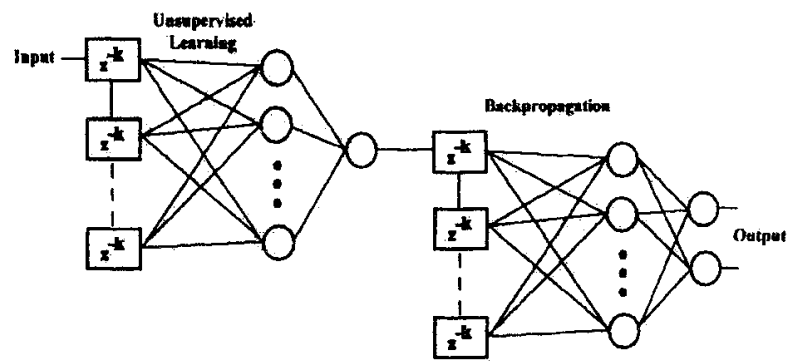

Fig. 7. Neural Network Configuration

The first component is a tap delay line to reconstruct the time series in a $\mathrm{D}$ dimensional space. The principal components of the reconstructed trajectory are extracted using Sanger's rule [6]. Sanger's rule is an on-line unsupervised learning rule that computes the principal components of a signal using the deflation method. Since here we want to filter the background noise the reconstruction of the input signal is done in a subspace of size $M$ such that $M \leq D$, where $D$ is the number of taps in the delay line and $M$ is the dimension of the PCA output space. The PCA outputs (eigenvalues) are summed to recombine the principal components that best describe the signal of interest. The result of the PCA is optimal filtering and data reduction.

The filtered time series is then fed into the time-delay neural network and distributed through a tapped-delay line with a memory depth of 200 samples. The MLP then uses the time-series history to discriminate between the data classes using as activation functions the hyperbolic tangent sigmoid function. The activations are then combined at the output layer into the network output which is a set of 2 linear PEs (one per target class). Thresholding is performed on the network outputs to acknowledge target results above the threshold and eliminate false detection.

The system is trained in two sweeps which are conveniently implemented in NeuroSolutions. First the PCA decomposition is obtained, the weights frozen, and only then the TDNN starts its training using the backpropagation algorithm. Momentum learning was utilized to speed up training. Training is stopped when the mean-square error in the cross-validation data set stops decreasing.

\section{Data Normalization and training/testing sets}


The input data is normalized so that the network trains on the waveform shape rather than on the relative amplitudes. The training set for detection consists of 100 target insect sounds (50 larvae, 50 adult), 100 false sounds (grain settling and external), and background noise from the grain separating each of the sounds. The test set for detection consists of 15 minutes of raw data, in which there are 537 adult sounds, 250 larvae sounds, and 469 false sounds. The test sets for classification consist of 100 adult insect sounds, 100 larvae insect sounds, 100 false sounds. The test sets for detection and classification problem each use sounds not found in the training sets.

\section{Construction of the desired response}

Figure 10 shows the target desired response which is a ramp that increases from zero to one with a slope of $1 /$ (sample width of the target signal). The width of a larvae sound is 100 samples, or $2.08 \mathrm{~ms}$. The width of all other sounds is 300 samples, or $6.25 \mathrm{~ms}$. A memory depth of 200 samples $(4.17 \mathrm{~ms})$ in the TDNN was found to be adequate to accurately classify all of the target sounds.

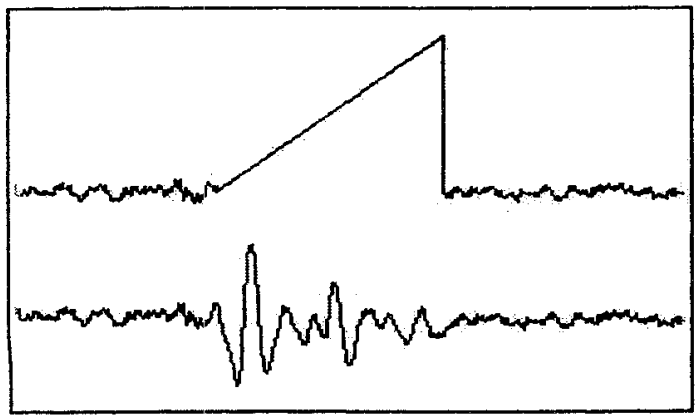

Fig. 8. Desired Response Construction

A desired response of zero during the background failed to train the network, since the network weights would converge to zero. Prediction of the background was usied between the target ramps so that the network would have nonzero values to train with. This approach was successful and eliminated the previous problem of null weights.

\section{Results}

\section{Detection Performance}

The receiver operating characteristic curve, or ROC-curve, is used to measure the detection performance of the neural network. ROC curves show the probability of detection versus the false alarm rate. The ROC curve is generated by passing the network output through a threshold detector that sweeps from the maximum to minimum values, in this case 1 and 0 , respectively.
Eight configurations of the neural network were tested for the detection of adult insects. The ROC curves for the detection of adult insects using the test set is shown in Figures 9-12 where the number of taps and hidden PEs is changed.

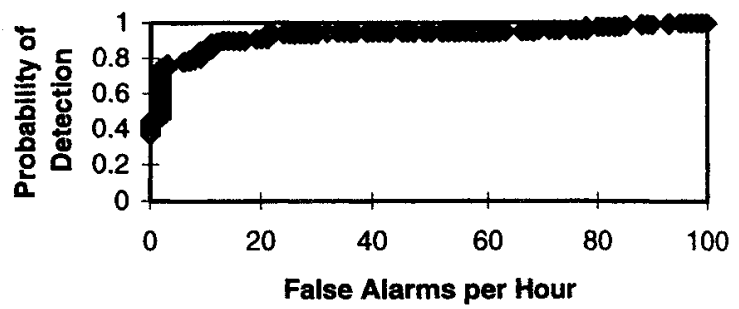

Fig. 9. ROC Curve for Network \#1 (50 taps, 4 sample delay, 5 PEs)

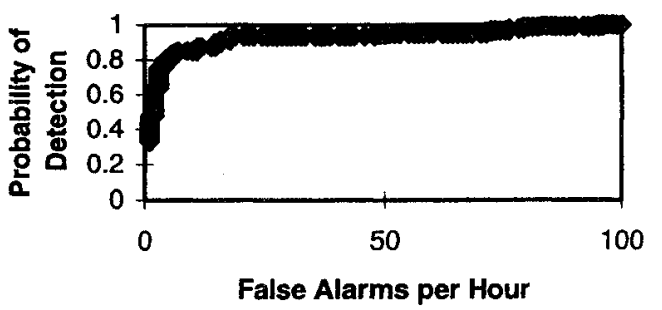

Fig. 10. ROC Curve for Network \#4 (50 taps, 4 sample delay, 20 PEs)

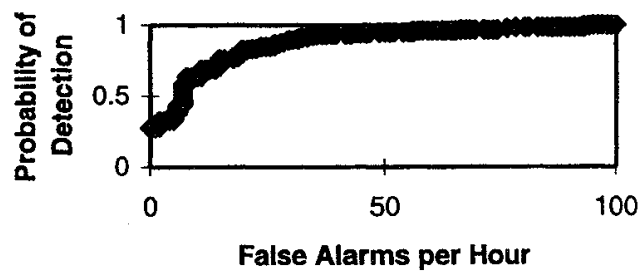

Fig. 11. ROC Curve for Network \#5 (25 taps, 8 sample delay, 5 PEs)

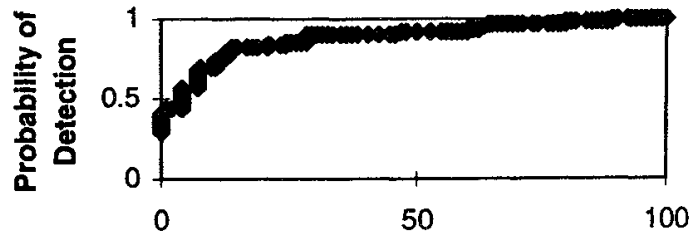

False Alarms per Hour

Fig. 12. ROC Curve for Network \#8 (25 taps, 8 sample delay, 20 PEs)

The best performer was combination of 50 taps and 20 PEs, which provided a number of false alarms per hour of 83 for $100 \%$ detection and 50 for $95 \%$ detection. The ROC curves for the detection of larvae insects are shown in Figures 13-24. 


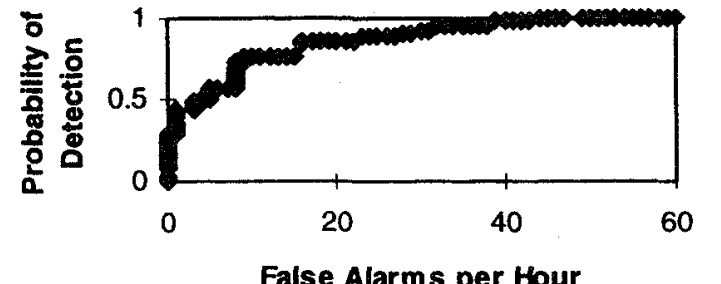

Fig. 13. ROC Curve for Network \#3 (50 taps, 4 sample delay, 5 PEs)

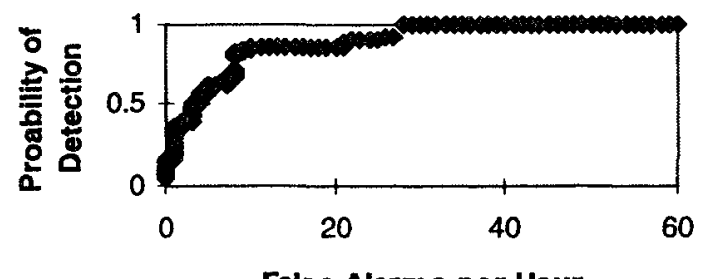

False Alarms per Hour

Fig. 14. ROC Curve for Network \#4 (50 taps, 4 sample delay, 20 PEs)

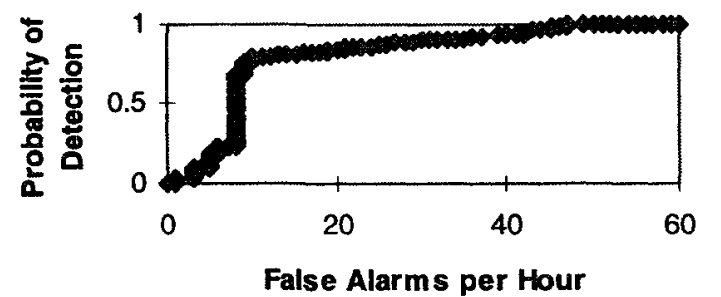

Fig. 15. ROC Curve for Network \#5 (25 taps, 8 sample delay, 5 PEs)

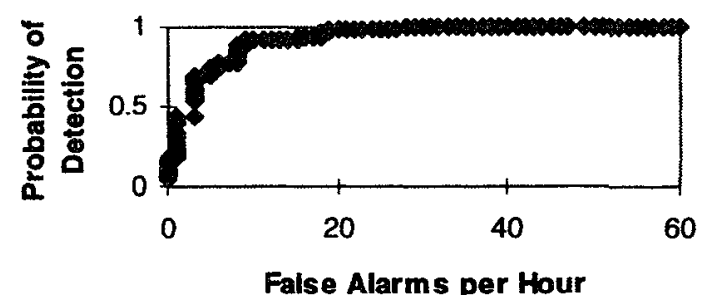

Fig. 16. ROC Curve for Network \#8 (25 taps, 8 sample delay, 20 PEs)

For the larvae, the performance is better and the detector with 50 taps and 20 PEs produced $100 \%$ detection with only 28 false alarms per hour ( 27 false alarms for $95 \%$ ).

\section{Classification Results}

Classification performance is measured using a confusion matrix. The three classes are larvae sounds, adult sounds, and false sounds. Eight network configurations were tested. The columns of the confusion matrix signify the class assignments as determined visually by a human. Machine performance is indicated by the rows. Agreement between the neural network classifier and the human classifier is shown on the diagonal of the matrix.

\begin{tabular}{l|lll} 
ANNHuman & larvae & adult & false \\
\hline larvae & 71 & 4 & 25 \\
adult & 22 & 69 & 10 \\
false & 7 & 28 & 65
\end{tabular}

Fig. 17. Confusion Matrix Network \#1: Taps: 50 Delay: 4 PEs: 5

\begin{tabular}{l|lll} 
ANNUHuman & larvae & adult & false \\
\hline larvae & 89 & 2 & 9 \\
adult & 7 & 84 & 9 \\
false & 4 & 14 & 82
\end{tabular}

Fig. 18. Confusion Matrix Network \#2: Taps: 50 Delay: 4 PEs: 10

\begin{tabular}{l|lll} 
ANNWHuman & larvae & adult & false \\
\hline larvae & 96 & 1 & 3 \\
adult & 2 & 93 & 5 \\
false & 2 & 6 & 92
\end{tabular}

Fig. 19. Confusion Matrix Network \#3: Taps: 50 Delay: 4 PEs: 15

\begin{tabular}{l|lll} 
ANNWHuman & larvae & adult & false \\
\hline larvae & 97 & 0 & 3 \\
adult & 1 & 98 & 1 \\
false & 2 & 2 & 96
\end{tabular}

Fig. 20. Confusion Matrix Network \#4: Taps: 50 Delay: 4 PEs: 20

\begin{tabular}{l|lll} 
ANNUHuman & larvae & adult & false \\
\hline larvae & 76 & 2 & 22 \\
adult & 2 & 81 & 17 \\
false & 22 & 17 & 61
\end{tabular}

Fig. 21. Confusion Matrix Network \#5: Taps: 25 Delay: 8 PEs: 5

\begin{tabular}{l|lll} 
ANNHuman & larvae & adult & false \\
\hline larvae & 87 & 2 & 11 \\
adult & 1 & 89 & 10 \\
false & 12 & 9 & 81
\end{tabular}

Fig. 22. Confusion Matrix Network \#6: Taps: 25 Delay: 8 PEs: 10

\begin{tabular}{l|lll} 
ANNWHuman & larvae & adult & false \\
\hline larvae & 92 & 2 & 6 \\
adult & 0 & 91 & 9 \\
false & 8 & 7 & 85
\end{tabular}

Fig. 23. Confusion Matrix Network \#7: Taps: 25 Delay: 8 PEs: 15 


\begin{tabular}{l|lll} 
ANNHuman & larvae & adult & false \\
\hline larvae & 93 & 2 & 5 \\
adult & 0 & 91 & 9 \\
false & 7 & 7 & 86
\end{tabular}

Fig. 32. Confusion Matrix Network \#8: Taps: 25 Delay: 8 PEs: 20

As we can expect the classification performance of the networks also changed according to the topology. We can see that the best performer is network \#4 with 50 taps and 20 hidden PEs. It seems that one needs the resolution to distinguish accurately among the three classes of sounds. These results also show that the system can detect grain settling sounds and can then improve upon the detection results shown earlier.

\section{Conclusion}

This paper presents a neural network capable of detecting and classifying insect sounds in a grain silo. Insect sounds are patterns in time so a dynamic network is needed to extract and classify the events from a time series. We do not know of any alternate methodology to detect insect sounds in grain sillos that performs as well as the TDNN described in this paper. But a lot of improvements can still be done to the existing design.

We would like to modify the neural network topology to include time recurrencies in the memory elements since they seem more appropriate than the ideal delays found in TDNN. We will also like to modify the frontend from a PCA to a self-organizing map or simply input the data to the dynamic neural network directly. Another interesting problem is to fine tune the training. Training a dynamic network means that the desired response must be designed in time. Here we used a predictive framework that seems to work but others may exist. Nevertheless, detecting and classifying sounds in grain silos seems possible with dynamic neural networks.

\section{Acknowledgment}

This research was partially funded by NSF grant ECS9510715 through a Research Experience for Undergraduates extension. We would like to thank the Agricultural Research Service of the USDA in Gainesville, Florida, for kindly supplying the data.
[1] D.K. Weaver, D. Shuman, R.W. Mankin, "Enhanced Performance of a Cross-Correlation Algorithm that Counts the Number of Infested Wheat Kernels in Grain Samples", Agricultural Research Service, USDA, 1996.

[2] Stored Grain Insects, Agricultural Research Service, USDA, 1986.

[3] Waibel A, et al., "Phoneme recognition using time delay neural networks", IEEE Trans. Acoust. Speech and Sign. Proc. , ASSP-37, 328-339, 1989.

[4] Zahalka A., Principe J., "Transient detection using neural networks: the search for the desired signal", in Advances Neural Information Proc. Systems 5, Ed. Hanson, Cowan, Giles, pp. 688-695, 1993.

[5] Thomas J., "Statistical Communication Theory", Wiley, 1974.

[6]Haykin S., "Neural networks: a comprehensive foundation", McMillan, 1994.

[7] NeuroSolutions User Manual, NeuroDimension, Inc. Gainesville, Florida, 1995.

\section{References}

